\title{
A Point-Wise Quantification of Asymmetry Using Deformation Fields: Application to the Study of the Crouzon Mouse Model
}

\author{
Hildur Ólafsdóttir ${ }^{1,2}$, Stephanie Lanche ${ }^{1,2,3}$, Tron A. Darvann ${ }^{2}$, \\ Nuno V. Hermann ${ }^{2,4}$, Rasmus Larsen ${ }^{1}$, Bjarne K. Ersbøll ${ }^{1}$, Estanislao Oubel ${ }^{5}$, \\ Alejandro F. Frangi ${ }^{5}$, Per Larsen ${ }^{2}$, Chad A. Perlyn ${ }^{6}$, Gillian M. Morriss-Kay ${ }^{7}$, \\ and Sven Kreiborg ${ }^{2,4,8}$ \\ ${ }^{1}$ Informatics and Mathematical Modelling, Technical University of Denmark, \\ Lyngby, Denmark \\ 2 3D-Laboratory, School of Dentistry, University of Copenhagen; \\ Copenhagen University Hospital; Informatics and Mathematical Modelling, \\ Technical University of Denmark, Copenhagen, Denmark \\ ${ }^{3}$ Ecole Supérieure de Chimie Physique Electronique de Lyon (ESCPE Lyon), France \\ ${ }^{4}$ Department of Pediatric Dentistry and Clinical Genetics, School of Dentistry, \\ Faculty of Health Sciences, University of Copenhagen, Copenhagen, Denmark \\ ${ }^{5}$ Computational Imaging Lab, Department of Technology - D.326, \\ Pompeu Fabra University, Barcelona, Spain \\ ${ }^{6}$ Division of Plastic Surgery, Washington University School of Medicine, St. Louis, \\ MO, USA \\ ${ }^{7}$ Department of Physiology, Anatomy and Genetics, Oxford University, Oxford, UK \\ ${ }^{8}$ Department of Clinical Genetics, The Juliane Marie Centre, \\ Copenhagen University Hospital, Copenhagen, Denmark
}

\begin{abstract}
This paper introduces a novel approach to quantify asymmetry in each point of a surface. The measure is based on analysing displacement vectors resulting from nonrigid image registration. A symmetric atlas, generated from control subjects is registered to a given subject image. A comparison of the resulting displacement vectors on the left and right side of the symmetry plane, gives a point-wise measure of asymmetry. The asymmetry measure was applied to the study of Crouzon syndrome using Micro CT scans of genetically modified mice. Crouzon syndrome is characterised by the premature fusion of cranial sutures, which gives rise to a highly asymmetric growth. Quantification and localisation of this asymmetry is of high value with respect to surgery planning and treatment evaluation. Using the proposed method, asymmetry was calculated in each point of the surface of Crouzon mice and wild-type mice (controls). Asymmetry appeared in similar regions for the two groups but the Crouzon mice were found significantly more asymmetric. The localisation ability of the method was in good agreement with ratings from a clinical expert. Validating the quantification ability is a less trivial task due to the lack of a gold standard. Nevertheless, a comparison with a different, but less accurate measure of asymmetry revealed good correlation.
\end{abstract}




\section{Introduction}

Crouzon syndrome was first described nearly a century ago when calvarial deformities, facial anomalies, and abnormal protrusion of the eyeball were reported in a mother and her son [1. Later, the condition was characterised as a constellation of premature fusion of the cranial sutures (craniosynostosis), orbital deformity, maxillary hypoplasia, beaked nose, crowding of teeth, and high arched or cleft palate. Heterozygous mutations in the gene encoding fibroblast growth factor receptor type 2 (FGFR2) have been found responsible for Crouzon syndrome 2 . Recently a mouse model was created to study one of these mutations (FGFR2 $\left.2^{\text {Cys } 342 \text { Tyr }}\right)$ 3].

Incorporating advanced small animal imaging techniques such as Micro CT, allows for a detailed examination of the craniofacial growth disturbances. A recent study, performing linear measurements on Micro CT scans, proved the mouse model applicable to reflect the craniofacial deviations occurring in humans with Crouzon syndrome 4 . Previously, we have extended this study to assess the local deformations between the groups by constructing a deformable shape- and intensity-based atlas of wild-type (normal) mouse skulls [5]. Statistical models of the deformation fields indicated that the skulls of Crouzon mice were more asymmetric than the wild-type skulls [6].

Asymmetry is highly relevant for the syndrome since the full or partial fusion of cranial sutures at either side of the skull and at different times causes the skull to grow asymmetrically. An accurate and localised assessment of asymmetry will improve surgery planning and treatment evaluation of children with Crouzon syndrome and other related diseases. In a previous study of ours, asymmetry in children with Deformational Plagiocephaly was measured using the ratio of left and right distances to a midpoint of a deformed symmetric template 7]. Another study on craniofacial malformations defined asymmetry as the deviation of the midsagittal surface with respect to the midsagittal plane 8. The estimation of asymmetry has also received some attention in the field of brain image analysis. In [9], structural asymmetry was defined as the significant deviation from a symmetric template. In [10] asymmetries were defined by warping grouprepresentative left and right hemispheric images to each other. In 11, voxel-wise structural and radiometric asymmetry was assessed in tumour brain images by defining a symmetry plane in each image and registering to the reflection.

We propose a novel asymmetry measure based on the deformation vectors resulting from nonrigid registration of a perfectly symmetric atlas image to a given subject image. The main advantage of the proposed method compared to [8], 10] and 11] is that it avoids defining a symmetry plane in each subject. This is important since defining such a symmetry plane in a skull affected by malformation is prone to errors. With respect to [9], where the left and right side relationship is ignored, the proposed approach compares the corresponding left and right deformations. We apply the asymmetry measure to locally quantify the asymmetry in Crouzon subjects and compare it to the control group. 


\section{Materials and Methods}

\subsection{Data Material}

Production of the $\mathrm{Fg} f r 2^{C 342 Y /+}$ and $\mathrm{Fg} f r 2^{C 342 Y / C 342 Y}$ mutant mouse (Crouzon mouse) has been previously described [3. All procedures were carried out in agreement with the United Kingdom Animals (Scientific Procedures) Act, guidelines of the Home Office, and regulations of the University of Oxford.

For three-dimensional (3D) CT scanning, 10 wild-type and $10 \mathrm{Fg} f r 2^{C 342 Y /+}$ specimens at six weeks of age (42 days) were sacrificed using Schedule I methods and fixed in $95 \%$ ethanol. They were sealed in conical tubes and shipped to the Micro CT imaging facility at the University of Utah. Images of the skull were obtained at approximately $46 \mu \mathrm{m} \times 46 \mu \mathrm{m} \times 46 \mu \mathrm{m}$ resolution using a General Electric Medical Systems EVS-RS9 Micro CT scanner.

\subsection{Quantification and Localisation of Asymmetry}

The proposed method makes use of a perfectly symmetric atlas created from healthy subjects. In a previous study, we have created a wild-type mouse atlas [5]. A midsagittal plane can then easily be determined using ear landmarks. Subsequently, a symmetric atlas was created by mirroring one half across the plane. In this way, correspondence between left and right voxels is known. To establish a left/right correspondence for any mouse image, the widely used B-spline-based nonrigid registration algorithm [12]13], was used to create correspondence fields between the symmetric atlas and a subject image. The transformation model of the nonrigid registration algorithm consists of a global (affine) and a local model (B-splines). For asymmetry calculations, only the local displacements are considered, in order not to make pose and scale differences affect the measure. Now, asymmetry can be calculated in each point of the deformed symmetric atlas.

The basic idea of the proposed asymmetry measure is to compare a displacement vector on one side to the corresponding displacement vector on the other side. More formally, asymmetry, $A_{P}$ of a point $P$ involves the comparison of the local displacement vector, $\mathbf{v}_{P}$ in point $P$ and the corresponding vector, $\mathbf{v}_{P^{\prime}}$ in point $P^{\prime}$ on the opposite side. The approach taken here is to use the mirrored vector $\mathbf{v}_{P^{\prime}}^{m}(x, y, z)=\mathbf{v}_{P^{\prime}}(-x, y, z)$. The absolute value of asymmetry is then defined by the magnitude of the vector difference,

$$
\left|A_{P}\right|=\left\|\mathbf{v}_{P}-\mathbf{v}_{P^{\prime}}^{m}\right\| \text {. }
$$

This, obviously, gives $A_{P}=0$ if the original vectors are perfectly symmetric. We define the absolute asymmetry at $P$ and $P^{\prime}$ to be equal. A sign is used to indicate whether the surface has expanded or depressed with respect to the point on the other side. Thus, $A_{P}$ and $A_{P}$ are defined by the following.

$$
\text { if } \begin{array}{r}
\mathbf{v}_{P}-\mathbf{v}_{P^{\prime}}^{m} \text { points outwards then } A_{P}=\left\|\mathbf{v}_{P}-\mathbf{v}_{P^{\prime}}^{m}\right\| \text { and } A_{P^{\prime}}=-A_{P} \\
\text { else } A_{P}=-\left\|\mathbf{v}_{P}-\mathbf{v}_{P^{\prime}}^{m}\right\| \text { and } A_{P^{\prime}}=-A_{P}
\end{array}
$$


This way of defining the direction of asymmetry is limited to surfaces extracted from the volume, since the surface normal is required. A volumetric measure could be obtained using the determinant of the Jacobian as in 11. Fig. 11illustrates the vectors involved in the calculation of the asymmetry measure.
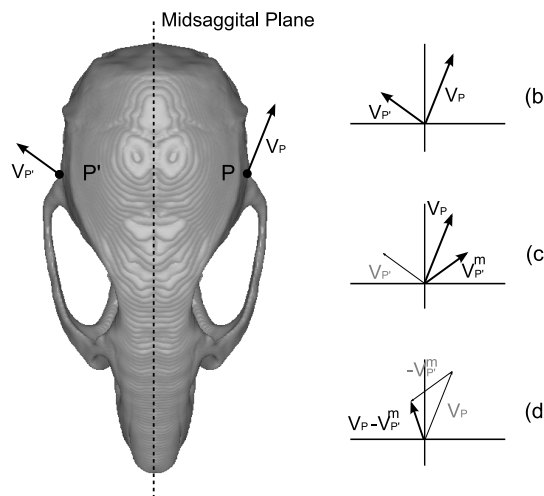

(c)

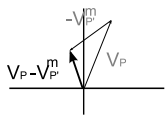

(d)

(a)

Fig. 1. Schematic figure of vectors involved in asymmetry calculation. (a) Displacement vectors shown on the symmetric atlas. (b) Displacement vectors placed in the origin. (c) Mirroring of $\mathbf{v}_{P \prime}$. (d) Difference vector. The magnitude of the difference vector defines the absolute asymmetry, $\left|A_{P}\right|$.

\section{Results and Validation}

Fig. 2 presents the results of the asymmetry computations in three example subjects from each group of mice. Fig. 3 provides a comparison of the groups in terms of absolute mean asymmetry.

Since the proposed method is based on results from image registration, the registration accuracy is essential for the method to be reliable. An extensive landmark validation using two sets of manual expert annotations as a gold standard was carried out in [5]. Landmark positions generated by the registration results were found to be non-significantly different from the gold standard and with significantly lower variance.

To evaluate the asymmetry detection itself, we consider that the proposed method both localises and quantifies asymmetry. In order to validate the two different aspects of the method, two approaches were taken. To evaluate the localisation ability of the method, a clinical expert rated nine different regions of anatomical interest on the skull of the original Crouzon surfaces. Those were (see Fig. 2(a,b)) the nose (viewed from above and below), zygoma, anterior skull, mid skull, posterior skull, basal maxilla, anterior cranial base and posterior cranial base.

The expert marked each region by 0 or 1 depending on whether the given region was symmetric or asymmetric, relatively. Similar ratings were obtained 

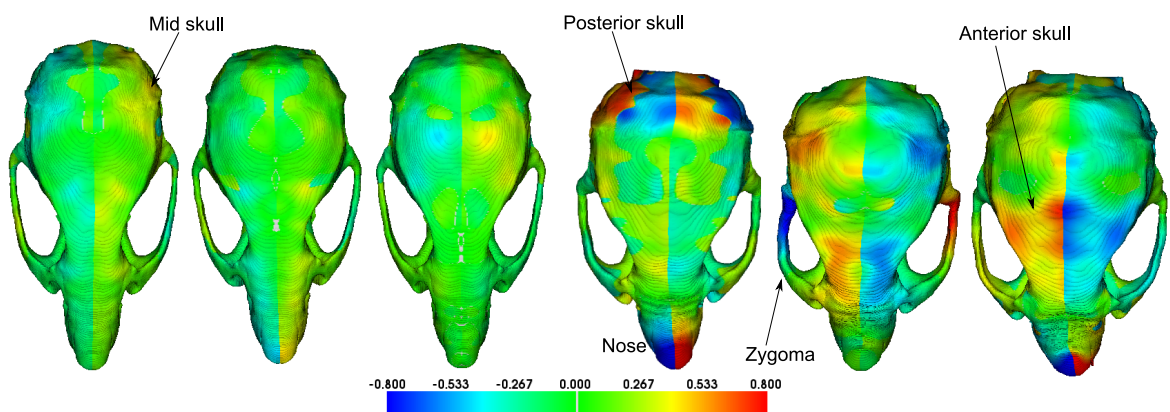

(a) Wild-type mice
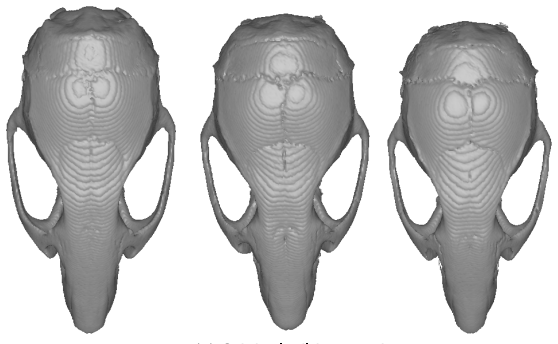

(c) Original wild-type mice

(b) Crouzon mice
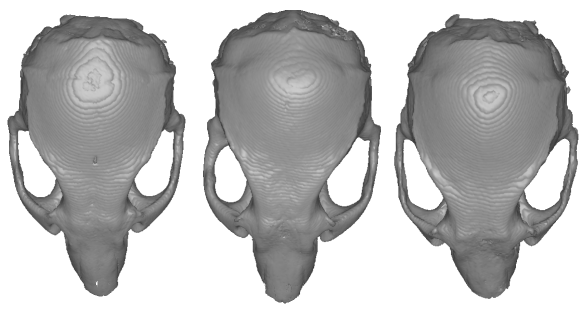

(d) Original Crouzon mice

Fig. 2. Example results for (a) three wild-type mice and (b) three Crouzon mice displayed on the deformed symmetric atlas. Asymmetry values shown in mm according to the colorscale. The scale ranges from blue (depressed) to red (expanded). Note that $A_{P}=-A_{P}^{\prime}$, i.e. each value on the left side has a corresponding negative value on the right side. For a visual comparison the corresponding original surfaces of (c) the wild-type mice and (d) the Crouzon mice are shown.

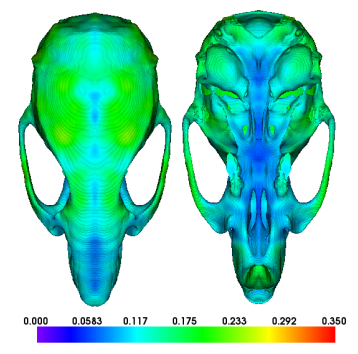

(a)

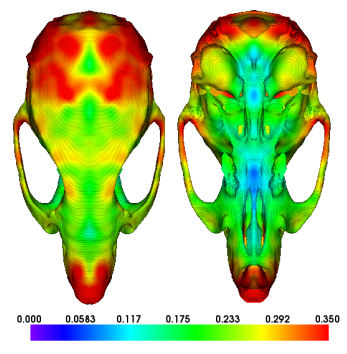

(b)

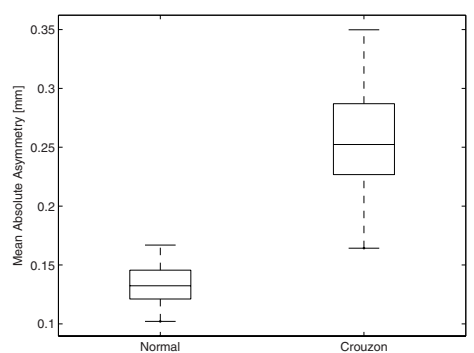

(c)

Fig. 3. Difference between groups. Mean absolute asymmetry of (a) wild-type and (b) Crouzon mice, displayed on the symmetric atlas in top and bottom views. Note that the colorscale is different from the one in Fig. 2. (c) Global mean absolute asymmetry in Crouzon mice and wild-type mice compared in a box plot.

from the automatic method where regions with $\left|A_{P}\right|>0.25 \mathrm{~mm}$ were marked by 1 and the remaining regions by 0 . Fig. 4(a) gives the number of regions where the automatic approach and the expert rating agreed. 
Validating the quantification ability of the asymmetry measure is more problematic, since a gold standard is not present. Here we take the approach to compare our method to a simple, crude measure of asymmetry. The original surfaces of all subjects were mirrored and closest point difference to the original surface was calculated. This method provides no point correspondences and is therefore not exact but the differences should correlate with the asymmetry values calculated by the proposed method. This is shown in Fig. 4(b).

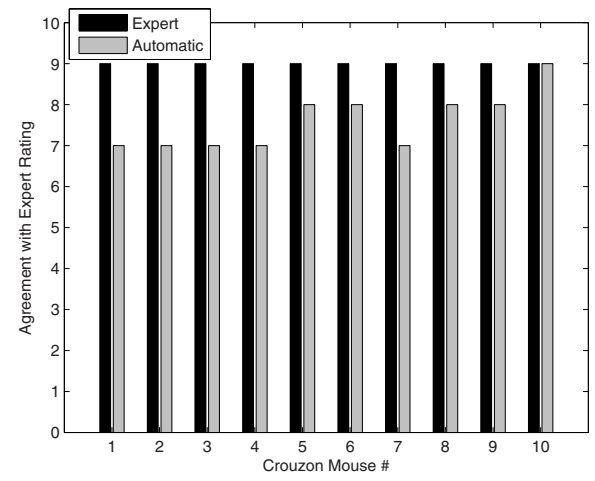

(a)

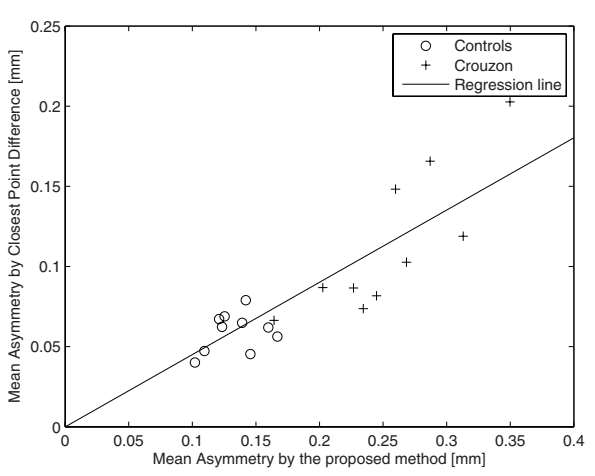

(b)

Fig. 4. a) Validation with respect to expert rating. The point of reference (or the gold standard) is defined by the expert (black bars), which indeed agrees with herself in rating of the nine regions for each mouse. The gray bars denote the number of regions where the automatic method agrees with the expert. b) Correlation of mean asymmetry using the proposed method vs. a closest point difference approach. $R^{2}=0.75$.

\section{Discussion}

Fig. 2(a) shows that the three wild-type mice have a few asymmetric regions of up to approximately $0.5 \mathrm{~mm}$ in absolute asymmetry. This is not obvious by an amateur inspection of these regions on the original surfaces in Fig. 2(c). The level of detection would be higher for a clinical expert. However, for the more asymmetric Crouzon mice, one can easily detect asymmetry on the original surfaces by the eye in the nose, zygoma and posterior skull (Fig. 2(d)). This is in good agreement with the automatic approach in Fig. 2(b). The asymmetry of the anterior skull detected by the proposed method is closer to the symmetry plane, and is harder to confirm by the eye.

Fig. 3 shows that the two groups differ considerably in terms of absolute asymmetry. From Fig. 3(a) and (b) we note that the trend is similar, i.e. asymmetry appears in similar regions, apart from the nose seen from above. However, as expected, the Crouzon mice have much higher degree of asymmetry. This is confirmed in the box plot in Fig. 3(c), which indicates that on average, Crouzon mice are more asymmetric than wild-type mice. This is also confirmed by a t-test 
on the absolute mean asymmetries (p-value of $10^{-6}$ ). This is exactly what was expected from the analysis, i.e. the premature fusion of the cranial sutures leads to an asymmetric skull.

Fig. 4(a) shows that the localisation ability of the automatic approach is in excellent agreement with the clinical expert. Most of the cases where the automatic method did not agree with the expert, were borderline, i.e. the asymmetry was just below or just above the selected threshold. The threshold of 0.25 approximately corresponds to the regions just turning into yellow in Fig. 2(b). Obviously, the choice of threshold is extremely important and the most correct way would probably be to use multiple parameter hypothesis testing to determine the threshold of significant asymmetry. For validation purposes, we believe that the fact that we are using the same threshold for all mice is important. For clinical practice it is perhaps even useful to be able to tune the threshold (or the range of the colorscale) with respect to the different experts' philosophical definitions of asymmetry.

Fig. 4(b) shows that the proposed method gives a relatively good correlation with a crude measure of asymmetry, with an $R^{2}$ of 0.75 . The fact that the correlation is not higher is understandable due to the closest point difference approach's lack of point correspondences. Nevertheless, it definitely shows that the two methods have the same trend and we believe that this is a good indication of that the quantification is correct.

\section{Conclusion}

Using the proposed asymmetry measure, Crouzon mice were seen to have significantly higher average asymmetry than the wild-type mice, confirming the clinical hypothesis. The localisation ability of the method was seen to correspond well with expert rating. It was considered more problematic to validate the quantification ability of the proposed method but a comparison to a crude asymmetry measure gave an acceptable correlation. In conclusion, a novel 3D asymmetry measure was developed, providing a detailed, surface map of asymmetry.

\section{Acknowledgements}

For all image registrations, the Image Registration Toolkit was used under Licence from Ixico Ltd.

\section{References}

1. Crouzon, O.: Une nouvelle famille atteinte de dysostose cranio-faciale héréditère. Bull Mem. Soc. Méd. Hôp. 39, 231-233 (1912)

2. Reardon, W., Winter, R.M., Rutland, P., Pulleyn, L.J., Jones, B.M., Malcolm, S.: Mutations in the fibroblast growth factor receptor 2 gene cause Crouzon syndrome. Nat. Genet. 8, 98-103 (1994) 
3. Eswarakumar, V.P., Horowitz, M.C., Locklin, R., Morriss-Kay, G.M., Lonai, P.: A gain-of-function mutation of fgfr2c demonstrates the roles of this receptor variant in osteogenesis. Proc. Natl. Acad. Sci. 101, 12555-12560 (2004)

4. Perlyn, C.A., DeLeon, V.B., Babbs, C., Govier, D., Burell, L., Darvann, T., Kreiborg, S., Morriss-Kay, G.: The craniofacial phenotype of the Crouzon mouse: Analysis of a model for syndromic craniosynostosis using 3D MicroCT. Cleft Palate Craniofacial Journal 43(6), 740-747 (2006)

5. Ólafsdóttir, H., Darvann, T.A., Hermann, N.V., Oubel, E., Ersbøll, B.K., Frangi, A.F., Larsen, P., Perlyn, C.A., Morriss-Kay, G.M., Kreiborg, S.: Computational mouse atlases and their application to automatic assessment of craniofacial dysmorphology caused by the crouzon mutation Fgfr2 ${ }^{\mathrm{C} 342 \mathrm{Y}}$. Journal of Anatomy 211(1), 37-52 (2007)

6. Ólafsdóttir, H., Darvann, T.A., Ersboll, B.K., Hermann, N.V., Oubel, E., Larsen, R., Frangi, A.F., Larsen, P., Perlyn, C.A., Morriss-Kay, G.M., Kreiborg, S.: Craniofacial statistical deformation models of wild-type mice and crouzon mice. In: Pluim, J.P.W., Reinhardt, J.M. (eds.) Medical Imaging 2007: Image Processing, SPIE, vol. 6512, p. 65121C (2007)

7. Lanche, S., Darvann, T.A., Ólafsdóttir, H., Hermann, N.V., Pelt, A.E.V., Govier, D., Tenenbaum, M.J., Naidoo, S., Larsen, P., Kreiborg, S., Larsen, R., Kane, A.A.: A statistical model of head asymmetry in infants with deformational plagiocephaly. In: Ersbøll, B.K., Pedersen, K.S. (eds.) SCIA 2007. LNCS, vol. 4522, pp. 898-907. Springer, Heidelberg (2007)

8. Christensen, G., Johnson, H., Darvann, T., Hermann, N., Marsh, J.: Midsagittal surface measurement of the head: an assessment of craniofacial asymmetry. Proceedings of the SPIE - The International Society for Optical Engineering 3661, 612-619 (1999)

9. Ashburner, J., Hutton, C., Frackowiak, R., Johnsrude, I., Price, C., Friston, K.: Identifying global anatomical differences: Deformation-based morphometry. Human Brain Mapping 6(5-6), 348-357 (1998)

10. Lancaster, J., Kochunov, P., Thompson, P., Toga, A., Fox, P.: Asymmetry of the brain surface from deformation field analysis. Human Brain Mapping 19(2), 79-89 (2003)

11. Joshi, S., Lorenzen, P., Gerig, G., Bullitt, E.: Structural and radiometric asymmetry in brain images. Medical Image Analysis 7(2), 155-170 (2003)

12. Rueckert, D., Sonoda, L.I., Hayes, C., Hill, D.L.G., Leach, M.O., Hawkes, D.J.: Nonrigid registration using free-form deformations: application to breast MR images. IEEE Trans. on Medical Imaging 18(8), 712-721 (1999)

13. Schnabel, J.A., Rueckert, D., Quist, M., Blackall, J.M., Castellano-Smith, A.D., Hartkens, T., Penney, G.P., Hall, W.A., Liu, H., Truwit, C.L., Gerritsen, F.A., Hill, D.L.G., Hawkes, D.J.: A generic framework for non-rigid registration based on non-uniform multi-level free-form deformations. In: Niessen, W.J., Viergever, M.A. (eds.) MICCAI 2001. LNCS, vol. 2208, pp. 573-581. Springer, Heidelberg (2001) 